\title{
PRECISIONES PARA EL EMPLEO DEL MÉTODO DEL MÓDULO DE BALASTO EN EDIFICACIÓN
}

\author{
(RECOMMENDATIONS FOR THE USE OF THE COEFFICIENT OF SUBGRADE REACTION)
}

Manuel J. Freire Tellado, Arquitecto

Dpto. de Tecnología de la Construcción. Univ. de A Coruña

ESPAÑA

Fecha de recepción: 29-VII-99

$480-4$

\begin{abstract}
RESUMEN
Actualmente las herramientas informáticas permiten el cálculo conjunto de la estructura y cimentación, lo que introduce un mayor grado de precisión en el dimensionado de ambas. Obviamente, esta técnica implica la modelización del terreno como requisito sine qua non, modelización que frecuentemente ha sido resuelta recurriendo al método del módulo de balasto.

En última instancia, la precisión del modelo de cálculo así generado va a depender del ajuste entre el comportamiento del terreno real y el representado por el modelo. El empleo del modelo dentro de sus límites de aplicación, así como la caracterización del suelo mediante un valor del módulo de balasto apropiado, son condiciones prioritarias para un cálculo correcto.
\end{abstract}

Desgraciadamente, pese a su trascendencia, el problema no recibe un tratamiento adecuado en los manuales. Este texto pasa revista a los condicionantes del método, recoge diversas formulaciones para la determinación del módulo a emplear en función del tipo de terreno y de cimiento $y$ reseña varias correcciones que diversos autores han propuesto para mejorar el modelo.

\section{SUMMARY}

It has only been recently that computers were available to analyze the combined of structure and foundation, introducing more accuracy in structural analysis. Obviously, this technique involves the soil modelling as necessary. The model of the coefficient of subgrade reaction has been often used for this task.

At last, the accuracy of the model depends on the agreement between predictions and soil behavior. The appropriate use of Winkler theory and the suitable determination of $k$ are essential conditions for a good analysis.

Unfortunately, manuals are not sufficiently concerned with this problem. The limits of this theory, some formulations for the determination of the $k$ values and a few improvements of the model are described in this paper.

\section{CONDICIONES DE BORDE DE LAS ESTRUCTURAS DE EDIFICACIÓN.LOS CIMIENTOS Y EL TERRENO.}

Toda estructura está, en último término, soportada exclusivamente por el suelo. Este aserto, tan sencillo como obvio, ha sido olvidado durante mucho tiempo en el campo del análisis de estructuras de edificación: durante mucho tiempo se han aplicado unas simplificaciones de las condiciones de contorno radicales y sólo recientemente se ha empezado a recoger algunas modelizaciones del terreno, si bien normalmente limitadas a ciertos tipos singulares de cimientos, como losas o vigas flotantes.

Así, frente a métodos de análisis de la superestructura muy sofisticados -matriciales $3 \mathrm{D}$, elementos finitos, cálculos 
en teoría de segundo orden- en los que se analizan numerosos efectos secundarios, se efectúa -en el ámbito de la edificación comercial- una simplificación sorprendente al suponer que la estructura e stá empotrada -o articulada- en su enlace con la cimentación. Ello equivale a despreciar tanto la deformabilidad de la cimentación como el efecto de la compresibilidad del suelo y, por tanto, de los asientos de la edificación y a la repercusión que este concepto tendrá en las leyes de esfuerzos resultantes.

El planteamiento anterior hunde sus raíces en tiempos pasados, con limitados recursos de cálculo, fundamentalmente manuales, en los que sólo un análisis por partes de la estructura hacía factible su tratamiento. Pese a la reconocida validez de esta técnica -con una sanción práctica evidente-, y dados los recursos de cálculo disponibles hoy en día, resulta defendible la tesis del estudio conjunto de la estructura, cimentación y terreno, que explicite las interacciones de las distintas partes: los logros exponenciales de la informática hacen hoy factible abordar el tratamiento analítico global del conjunto estructura-cimiento-suelo. Es más, la modelización de parte del terreno de cimentación es norma usual en el estudio de presas y grandes obras de ingeniería.

El interés de tratamientos de este estilo va más allá del campo meramente teórico: ya nos advirtieron algunos investigadores sobre la dificultad conceptual de admitir las condiciones de borde referidas cuando se cuantifican los desplazamientos y giros que se producen en las piezas con las distintas condiciones de borde -empotramiento, articulación, apoyos deslizantes- de los apoyos y la dificultad de garantizar que las deformaciones que significarían el cambio de las condiciones de borde no se produzcan en la cimentación construida, garantizando así la validez de las hipótesis de cálculo supuestas.

Problema relevante, por cuanto asientos y rotaciones de la cimentación provocan serias variaciones en las leyes generales de esfuerzos de la estructura, significativamente en la redistribución de los esfuerzos flectores y axiles de pilares. Por fortuna, ciertos programas comerciales de cálculo de estructuras muy sofisticados incluyen entre sus opciones la posibilidad de modelización del terreno y del cimiento, analizado como placa sobre apoyos elásticos, solución que permite resolver con corrección, tanto el dimensionado de estos elementos, como las leyes de esfuerzos de la estructura, traduciéndose en un significativo incremento de la precisión analítica $y$ conceptual del modelo.

El segundo aspecto que justifica la modelización del cimiento y del terreno es, tal y como se ha avanzado en el párrafo anterior, el del dimensionado de las estructuras de cimentación: -de una parte se continúan calculando los esfuerzos a que éstas se encuentran sometidas como si se tratase de sólidos rígidos, sólidos rígidos que provocan un reparto lineal de las tensiones de respuesta del terreno, sólo posible si se considera la cimentación como infinitamente rígida [1]. Es esto una carga heredada de épocas pasadas, por cuanto las estructuras de cimentación son hoy mayormente flexibles, habiendo disminuido mucho asimismo la rigidez de la superestructura, consecuencia del incremento de luces y disminución de secciones de las piezas [2].

-por otro lado, las estructuras de cimentación superficiales-zapatas- se suelen estudiar como piezas aisladas, ignorando que la mayoría de las veces se encuentran enlazadas con nervios con otras partes de la cimentación, formando verdaderas 'zapatas combinadas de geometría variable'.

-el comportamiento no lineal y dependiente del tiempo del terreno complica aún más el análisis de éstas -se han hecho intentos de considerar la respuesta temporal a partir de la Teoría de Consolidación de Terzaghi [3] -.

Obviamente, la bondad del tratamiento de estos elementos recae en una modelización del suelo adecuada, convirtiéndose ésta en el primer problema a resolver. Los programas a los que se ha hecho referencia anteriormente resuelven la modelización mediante el empleo del Método del Módulo de Balasto o de Winkler, método que, además de su sencillez, cuenta con aval experimental referido al conjunto edificio-cimentaciónterreno aunque, lamentablemente, aún bastante reducido. Presenta, además, la ventaja fundamental de su fácil implementación informática: caracteriza el terreno exclusivamente por el valor de un parámetro, el módulo de balasto, dependiente del tipo de terreno, pero independiente de la formulación que permite su obtención.

Lógicamente, este tratamiento del terreno puede plantear cierta discusión: es un método que no se ajusta a la realidad, con problemas de todos conocidos -que luego se revisarán-; pero es cierto también que, en el ámbito del estudio, métodos más precisos como el modelo hiperbólico-elástico no lineal-no aportan mayor precisión en los resultados. Esto es así por cuanto que, en edificación convencional, las tensiones transmitidas al terreno no son altas: los coeficientes de seguridad usualmente empleados en cimentaciones se traducen en tensiones que rara vez superan el tercio de la carga de hundimiento del terreno -ya que la tensión admisible engloba ese doble factor de seguridad suficiente frente a hundimiento y frente a asientos excesivos-por lo que los cimientos se encuentran en la parte baja de la rama tensión/deformación, que es en todos los modelos propuestos bien una recta o bien sustituible por una recta sin error apreciable. En palabras de Jiménez Salas [4]. 
-Sin embargo, en muchos suelos, antes de llegar a este punto [rotura] existen estados en los que es muy aproximado suponer que hay una correspondencia entre las deformaciones y las fuerzas aplicadas.

Abundando en esta idea, señala en otro punto -[5] pág. 3-, que si el coeficiente de seguridad al hundimiento es del orden de 3, el estado tensional del suelo parece corresponder bastante bien con el deducido de la hipótesis del suelo perfectamente elástico -véase Gráfico 1 tomado de la referencia [6], pág. 323- .

Terzaghi [6] señala que la hipótesis de que el valor del módulo de balasto es, en cada punto, independiente de la presión de contacto, $p$, es, de acuerdo con las relaciones mostradas por el diagrama anexo -Gráfico 1- es aproximadamente válida solamente para valores de $p$ que no superen la mitad de la capacidad portante última $p_{u}$.

El Dr. John T. Christian [7] proporciona una recopilación de resultados de estudios de campo de diferentes autores sobre là Interacción Suelo-Estructura, con un saldo positivo para el método, pero con grados de ajuste muy diferentes, desde excelente -obtenido por Whitman et al. ([8], 1971)- hasta otros menos ajustados de De Simone y Gould ([9], 1971), con movimientos reales dobles de los predichos -aunque correctos en forma- sobre un soporte de arcilla azul de Boston -un descargo evidente-. Los resultados de estos estudios se pueden consultar en la referencia indicada.

Empero, la bibliografía sobre el tema apunta a que no todos los terrenos admiten una modelización igualmente satisfactoria con el método del Coeficiente de Balasto. Dentro de los tipos de suelos excluidos de la aplicación de métodos lineales se encuentran las arcillas normalmente consolidadas. Labor fundamental del técnico será, por tanto, discernir en qué situación el modelo resulta aplicable y cuándo no lo es.

Parece también dudosa la aplicación del método para el estudio de cimentaciones no superficiales -ya que las expresiones propuestas para la determinación de $k$ se corresponden con condiciones superficiales o casi superficiales, que minimicen el error de suponer un valor del Módulo de Young nulo en superficie del terreno- por lo que existen dudas sobre la adecuación del método para el estudio de cimentaciones de torres [7]. La hipótesis -se reseñará más adelante- de un valor de $E=0$ en superficie parece excluir también la representación de terrenos sometidos a un nivel previo de tensiones importante -preconsolidaciones-.

De todas formas, el problema anterior de la interacción con el terreno en el caso de cimentaciones profundas se ve reducido, porque normalmente las cargas son transmitidas

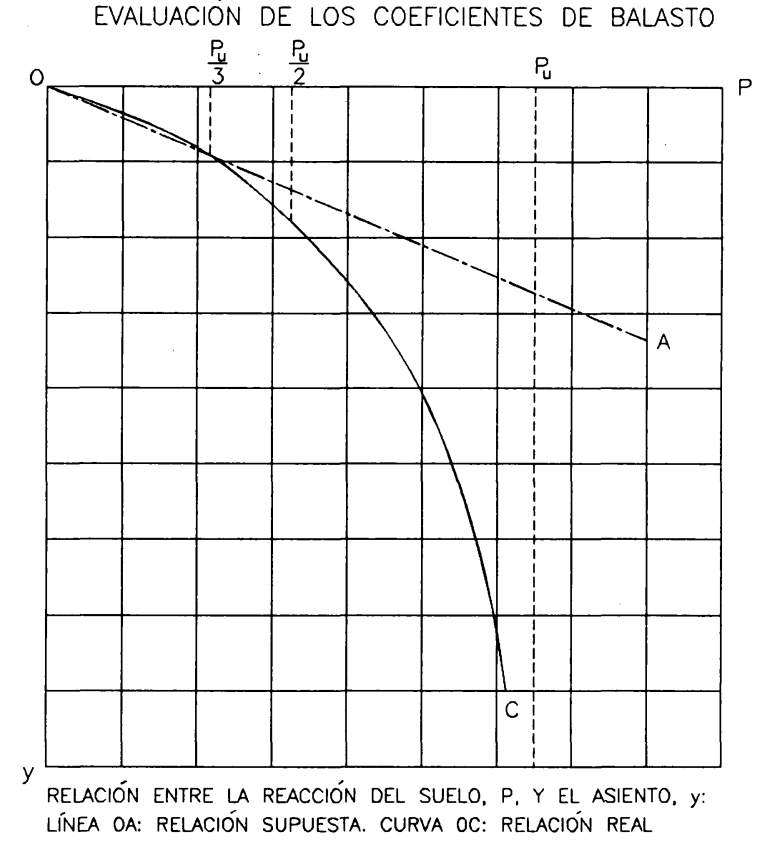

Gráfico I

a formaciones rígidas $\mathrm{y}$, por tanto, experimentan deformaciones muy pequeñas que no afectan significativamente a la estructura.

La discusión anterior pretende hacer patente la necesidad de que el técnico conozca mínimamente las condiciones de uso del modelo que va a utilizar, para decidir sobre si resulta o no aplicable al caso que analiza. Más aún, el valor del coeficiente de balasto a emplear no resulta trivial en absoluto, dependiendo de diversos factores -entre ellos el tamaño del cimiento y la profundidad del plano de apoyo-. Por desgracia, la información que se encuentra en los manuales de los programas referidos no es muy abundante en ninguno de estos aspectos.

\section{EL MÉTODODEL COEFICIENTE DE BALASTO}

El Método del Módulo de Balasto es conocido por el nombre de Método de Winkler -ya que fue en 1867 su introductor [10]-y que sirvió de base para el estudio, hoy ya clásico, de Zimmermann sobre el análisis de rieles de ferrocarril sobre traviesas [11].

Es bien sabido que es un modelo que no se ajusta a la realidad experimental del terreno, si bien los resultados que históricamente ha proporcionado están avalados por la experiencia -véase Dr. John T. Christian [7]-, factor de considerable importancia en un análisis como el que se aborda. Tal y como se ha señalado anteriormente, esto puede ser debido a que, en edificación convencional, las tensiones transmitidas al terreno son de pequeña entidad -limitadas tanto por el coeficiente de seguridad, suficiente 
frente a hundimiento, como por la necesidad de limitar los asientos para que no resulten excesivos- y, en esta zona, los valores responden con cierta aproximación a los que resultan de este modelo.

El método parte de la hipótesis de que el asiento producido en un punto es proporcional a la presión a la que está sometido, transmitida por la cimentación. Analíticamente,

$$
p=k_{\mathrm{s}} \cdot y
$$

donde $p$ representa la presión transmitida al terreno, $y$-ós- el asiento experimentado $k_{s}$ el factor de proporción entre ambos, conocido con el nombre de Módulo de Reacción, Coeficiente de Reacción [12], Coeficiente de Reacción de la Subrasante [6], Coeficiente o Módulo de Balasto, con unidades de $\mathrm{kg} / \mathrm{cm}^{3}$.

La formulación se completa para los casos de carga lineal $q$ repartida sobre un ancho $b$ y para carga concentrada $P$ actuando sobre una placa de área $A$ como sigue:

$$
q=k_{s} \cdot b \cdot y=k \cdot y \quad P=k_{s} \cdot A \cdot y 0 K \cdot y
$$

donde las unidades de $k y K$ son variables para permitir la homogeneidad de la fórmula.

Como demostró Gibson en sus estudios de 1967 y 1974 [13], el Método de Winkler es un caso particular de un Modelo de Suelo Elástico, Lineal, para un suelo Isótropo y Heterogéneo, con Módulo de Young Linealmente Variable en Profundidad. Las particularidades del modelo dentro de este grupo general se estriban en que considera un valor nulo en superficie para el Módulo E, y se supone que el terreno es incompresible, $\mu=0,5$.

Se puede representar la variación lineal del módulo de Young en función de la profundidad $z$ como [14]

$$
E(z)=E(0)+\lambda \cdot z
$$

donde $E(0)$ representa el valor del Módulo de Young en superficie y $\lambda \cdot z$ el incremento debido a la profundidad. De acuerdo con lo dicho anteriormente, el método considera que el módulo de Young varía con la profundidad de acuerdo con la ley

$$
E(z)=\lambda \cdot z
$$

Y el Módulo de Balasto correspondiente, de acuerdo con la expresión siguiente, responde a la relación

$$
K=\lambda / A \text {, que si } \mu=0,5 \text { se convierte en } K=2 / 3 \cdot \lambda
$$

John T. Christian [7] indica que Masaal'skii (1964) [15] y Ward et al. (1968) [16] han encontrado referencias experimentales que ratifican esta demostración.
La problemática del planteamiento del método queda así de manifiesto: por una parte considera una relación elástica lineal entre tensiones y deformaciones, regida por un módulo de Young con un valor nulo en superficie y que varía linealmente en la profundidad, suponiendo además que el terreno es incompresible.

Lo referente a la relación elástica lineal ha sido objeto de un comentario anterior. El módulo de deformación en terrenos homogéneos es, evidentemente, creciente con la profundidad, pero que este crecimiento se produzca por correlación lineal es, cuanto menos, discutido entre los expertos.

El valor nulo del módulo de Young en superficie es incorrecto incluso para suelos muy flojos. No entremos en la consideración del efecto de la preconsolidación del terreno, en el que para cargas inferiores a la de consolidación el asiento sería despreciable y por tanto, problemática la defensa del modelo.

Sin embargo, ensayos de laboratorio con placa de carga han demostrado que mientras las cargas no superen un cierto límite, los asientos son proporcionales a las cargas aplicadas -hipótesis general del método-. Se sabe empero que el asiento de una gran cimentación es muy diferente del encontrado para una pequeña superficie a la misma presión unitaria, razón que justifica una serie de correcciones introducidas al método y que incluso puede llegar a inhabilitarlo para ciertas soluciones y a partir de ciertas dimensiones de los elementos.

De todas formas los rasgos que se acaban de señalar no son más que particularidades de la hipótesis fundamental del método que considera el terreno como un fluido -incompresible-, despreciando parámetros tales como el ángulo de rozamiento interno y la cohesión, y con el agravante de que los terrenos no se comportan como líquidos. Esto se remite a la hipótesis fundamental del método: solamente el terreno bajo el cimiento experimenta deformaciones.

Se sabe que el asiento de los diferentes puntos del terreno depende del reparto de presiones en el suelo bajo las distintas cargas aplicadas. Sin embargo son conocidos desde los ensayos de Föppl de 1922 - citado en la referencia [5], pág 4.-, gran variedad de terrenos para los que resulta admisible la hipótesis de que el suelo se deforma únicamente bajo la zona directamente cargada.

Y esto, que es una falacia conceptual, tiene sin embargo la importancia que permite ignorar la superposición de los distintos bulbos de presiones de los cimientos del edificio -o de otros cimientos existentes- dentro de ciertas magnitudes proporcionando unos resultados que, históricamente, han demostrado un grado de fiabilidad aceptable. 
Fiabilidad que se explica -al menos parcialmente-porque, como es sabido -si se llama $b$ a la dimensión mayor del cimiento-, a una profundidad de $1,5 \cdot b$-de forma más precisa, a una profundidad variable entre $b$ y $2 \cdot b$-, la tensión transmitida al terreno es del orden de $0,1 \cdot \sigma$, de forma tal que para las dimensiones de cimientos usuales en cimentación no suele haber problemas de superposición. Estudios recientes han mostrado que para profundidades del orden de $4 \cdot b$ no suele haber modificación tensional del terreno o bien ésta es insignificante.

Otra razón que justifica el interés en el empleo del método es introducir coherencia con algunos de los métodos de Cálculo de Zapatas, que plantean implícitamente el método del coeficiente de balasto -explícitamente en el caso del cálculo de zapatas de medianería y esquina-. Sin embargo, lo hacen aplicando la hipótesis de cimiento rígido, que conduce a una simplificación excesiva dados los métodos actuales disponibles.

Se debe recordar que el método, tal y como se propuso inicialmente, descuida el efecto de factores tan importantes como:

- Naturaleza del Terreno en Superficie y sobrecarga aplicada.

- Prófundidad del Plano de Cimentación.

- Relación de Profundidad Plano Cimentación/Ancho de Zapata.

- Posición Relativa del Nivel Freático.

- Presencia de Capas Compresibles a Profundidad que puedan ser afectadas.

- Forma y Dimensiones de la Cimentación.

- Nivel de Cargas Aplicado.

- Nivel de Tensiones en el Terreno.

Hoy, el Coeficiente de Balasto se hace depender de:

1. Módulo de Compresibilidad $E_{s}$ bajo el cimiento.

2. Forma y dimensiones de la cimentación -rectangular, cuadrada, circular...-

3. Proporción entre las dimensiones del cimiento y cargas aplicadas.

\section{Rigidez Relativa del cimiento.}

\section{Nivel de Cargas: Cargas Vivas/ Cargas Muertas.}

En el planteamiento del método se han llegado a introducir correcciones -como, por ejemplo, la referencia [17]- que garantizan cierta continuidad en la deformación del soporte -suelo-, de tal forma que se garantiza en el modelo alguna continuidad del soporte. La referencia [18] puede ser valiosa en este tema.

\section{EXPRESIONES ANALÍTICAS DEL MÓDULO DE BALASTO}

De acuerdo con la hipótesis general del método, en pequeñas superficies y para placa circular se introduce, siguiendo a Caquot [19], la hipótesis de que el coeficiente de balasto es inversamente proporcional al diámetro de la placa empleada:

$$
d \cdot K_{b}=d^{\prime} \cdot K_{b^{\prime}}
$$

donde $d$ y $d$ 'son los diámetros de la placa de ensayo y $K_{b}$ y $K_{b}$, son los módulos de balasto correspondientes a esos cimientos determinados. Se admite igualmente que el valor correspondiente a una placa de carga cuadrada equivale al de una placa circular de igual área. Esta hipótesis admite implícitamente que, para el rango de dimensiones de las placas de carga -de 30 a $75 \mathrm{~cm}$ - el asiento producido es proporcional al tamaño de la cimentación.

Las relaciones anteriores son especialmente útiles para correlacionar resultados experimentales, normalmente derivados de ensayos realizados con las placas de diámetros normalizados 34 y $75 \mathrm{~cm}$ o con placa cuadrada de $30 \mathrm{~cm}$ de lado, de tal forma que la expresión anterior se convierte en $34 \cdot K_{34}=75 \cdot K_{75}$ para las placas circulares, admitiéndose además que $K_{34}=K_{30^{\prime}}$, siendo este último el valor para là placa cuadrada de lado 30 .

La expresión anterior se admite para todo tipo de terrenos y para pequeñas dimensiones de la faja de carga. Cuando las dimensiones son las usuales en cimientos de edificación precisa de ciertas correcciones, algunas de ellas ya establecidas por Terzaghi en 1955 [6], para desterrar la errónea concepción, ampliamente extendida entre los ingenieros, de que el Módulo de Balasto depende exclusivamente de la naturaleza del subsuelo -[6], pág. 300-.

En la referencia anterior, Terzaghi fundamenta la formulación en una reflexión sobre el tamaño de los bulbos de presiones y los asientos producidos. Posteriormente [3] justifica las expresiones que evalúan los valores del Módulo de Balasto a partir del estudio de los asientos de zapatas corridas de diferentes dimensiones trabajando a presión constante sobre arenas y arcillas homogéneas. Terzaghi aproximó la variación del asiento ocurrido en las zapatas a partir del experimentado por una placa de carga cuadrada de $30 \mathrm{~cm}$-si bien las dimensiones fueron expresadas en pies en la formulación original, se han reescrito adaptadas al Sistema Internacional- como:

$$
\begin{aligned}
& s_{c}=s_{30} \cdot \frac{b_{c}}{30} \quad \text { (arcillas duras) } \\
& s_{c}=s_{30}\left(\frac{2 \cdot b_{c}}{b_{c}+30}\right)^{2} \quad \text { (arenas) }
\end{aligned}
$$


donde $s_{30}$ es el asiento experimentado por la placa de $30 \mathrm{~cm}, s_{c}$ es el asiento del cimiento y $b_{c}$ es el ancho del cimiento. De acuerdo con la ecuación inicial, y dado que la presión transmitida es constante, es inmediato obtener:

$$
\begin{aligned}
& k_{c}=k_{30} \cdot \frac{30}{b_{c}} \text { (arcillas duras) } \\
& k_{c}=k_{30}\left(\frac{b_{c}+30}{2 \cdot b_{c}}\right)^{2} \quad \text { (arenas) }
\end{aligned}
$$

que son las ecuaciones clásicas propuestas por Terzaghi en 1955 [6], y que se recogen en [3] para zapatas $o$ cimentaciones corridas, donde $k_{30}$ es el valor del balasto para placa de $30 \mathrm{~cm}$ y $k_{c}$ es el correspondiente al cimiento. Sobre ellas John T. Christian -[7] pág. 151-indica que estas ecuaciones son aplicables exclusivamente a cimentaciones superficiales y casi superficiales, por lo que su aplicación a cimentaciones de edificios altos es dudosa.

Terzaghi en referencia [6], pág. 315, añade además:

$$
k_{c}=k_{30}\left(\frac{\alpha+0,5}{1,5 \cdot \alpha}\right) \quad \text { siendo } \alpha=\frac{l_{c}}{b_{c}} \begin{aligned}
& \text { (placas cuadradas } \\
& \text { sobrecomprimidas) }
\end{aligned}
$$

expresión válida para una placa cuadrada de 1 pie $(30 \mathrm{~cm})$, siendo $l_{c}$ la longitud del cimiento. El propio autor aclara que, para zapatas aisladas, zapatas continuas y vigas flotantes, las expresiones anteriores son válidas siempre que la presión de contacto no supere la mitad de la capacidad portante última del subsuelo; mientras que para losas sometidas a cargas concentradas lo son mientras que ninguna de las cargas concentradas supere la mitad de la capacidad portante última de la zapata equivalente de radio $\mathrm{R}$-la zapata circular equivalente es un concepto que acuña Terzaghi para el cálculo del módulo de balasto en losas con cuyo diámetro se entraría en las fórmulas anteriores-.

Empero la expresión anterior, en las referencias [3] y [6] el autor señala que no hay mayor diferencia entre el asentamiento de zapatas continuas y cuadradas de igual ancho $B$, por lo que las expresiones anteriores resultan también de aplicación para este último caso -idea que ha sido rebatida por la bibliografía posterior, como se ve seguidamente-.

Autores posteriores [21] [12] han adaptado esta expresión generalizándola para cualquier ancho de cimiento $\mathrm{y}$ expresándola en el sistema internacional de unidades

$k_{c}=k_{30} \frac{2 . \alpha+1}{3 . \alpha} \frac{b_{30}}{b_{c}} \quad$ siendo $\alpha=\frac{l_{c}}{b_{c}} \begin{aligned} & \text { (arcillas } \\ & \text { preconsolidadas) }\end{aligned}$

Nótese asimismo que se está diferenciando la expresión del Módulo de acuerdo con el tipo de terreno e introduciendo una corrección en razón al tamaño del cimiento, ya que, además, la constante de muelle de
Winkler depende fuertemente de la geometria de la carga.

Terzaghi, empero, recomienda el método de Winkler para el cálculo de momentos, pero no para asientos, debido a las simplificaciones que el método contiene. El propio Terzaghi, en su exposición de 1955 -[6] pág 301- ha señalado dos simplificaciones apriorísticas:

1. El Módulo de Balasto es, en cada punto, independiente de la presión de contacto.

2. El Módulo de Balasto tiene el mismo valor en todos los puntos de la superficie de contacto.

La primera hipótesis se ha analizado al principio del capítulo, demostrándose su aproximada validez dentro de un rango de valores de presión sobre el terreno, siempre inferior a la mitad de la presión de hundimiento.

La segunda de ellas se ha comprobado relevante en análisis sobre base perfectamente elástica, en los que se ha demostrado que el Módulo de Balasto depende, en cierta proporción, de la rigidez a flexión de la viga. A ambas simplificaciones, estudios posteriores, han asignado factores de corrección.

Estudios e investigaciones sucesivas han generalizado y reescrito parcialmente estas expresiones en la forma que sigue, donde $b_{0}$ representa el lado de la placa de carga [20].

$$
k_{c}=k_{0} \cdot \frac{b_{0}}{b_{c}} \quad \begin{aligned}
& \text { (arenas sueltas o muy sueltas, } \\
& \text { limos, arcillas) }
\end{aligned}
$$

Suelo Poco o No Coherente:

$$
k_{c}=k_{0}\left(\frac{b_{c}+b_{0}}{2 \cdot b_{c}}\right)^{2} \quad \text { (arenas densas) }
$$

Suelo Muy Coherente:

$$
k_{c}=k_{0}\left(\frac{b_{c}+b_{0}}{3 \cdot b_{c}}\right)^{2} \quad \text { (arenas compactas) }
$$

Para algunos autores-[12] pág.310-, cuando el ancho $b=1 m$, se acepta:

$$
k_{c}=k_{0}\left(0,25+\frac{0,15}{B}\right)
$$

con B en metros-.

Otros autores -[21], pág. 207; [12], pág. 310- recogen, además, la última de las expresiones de Terzaghi citadas, 
generalizándola para cualquier ancho de cimiento y expresándola en el sistema internacional de unidades

$k_{c}=k_{30} \frac{2 . \alpha+1}{3 . \alpha} \frac{b_{30}}{b_{c}} \quad$ siendo $\alpha=\frac{l_{c}}{b_{c}} \quad \begin{aligned} & \text { (arcillas } \\ & \text { preconsolidadas) }\end{aligned}$

Resulta preocupante, por su falta de rigor, la propuesta de algunos conocidos programas comerciales de cálculo de estructuras de extender el empleo de la expresión anterior al campo de las arcillas.

Lamodelización del terreno se vuelve, con las correcciones anteriores, un poco más específica.

El método ha ido modificando las expresiones que correlacionan los valores de los Módulos de Balasto para distintos tipos de suelo y distintas situaciones y dimensiones de cimiento, corrigiendo aquellas discordancias observadas entre su aplicación teórica y las comprobaciones de carácter experimental (infravaloración de asientos, exceso esfuerzos).

Dentro de este planteamiento se han desarrollado estudios tendentes a la determinación del Módulo en los casos de Zapatas Unidas por Vigas de Atado -recogidos en la referencia [22]-:

Se exponen a continuación diferentes métodos de estimación del Módulo de Balasto propuestas por diferentes autores, indicando el año de su proposición y la referencia.

\section{MÉTODOS DE ESTIMACIÓN DEL MÓDULO DE BALASTO}

\section{A partir de Tabulaciones Publicadas:}

[17] $A C I-336.2 R-88$ : señala las siguientes:

Terzaghi, 1955 [6]. Bowles, 1974, 1982, 1984 [23][24][25].

Dept. of Navy, 1982 [26]. Kramrish, 1984 [27].

\section{REFERENCLASESPAÑOLAS:}

[28] Calavera, Cálculo de Estructuras de Cimentación. Tablas GT-8 y GT-9

[29] Jiménez Salas, Geotecnia y Cimientos III. Capítulo 1. Tabla 1.1

\section{A partir de Ensayos en Placa de Carga:}

[17] $A C I-336.2 R-88$ : cita, entre otros:

Terzaghi, 1955 [6] -señalados anteriormente-. Sowers, 1977 [30]:

$$
k=k_{0}\left(\frac{b_{0}}{b_{m}}\right)^{n} \quad 0,5 \leq n \leq 0,7
$$

(c) Consejo Superior de Investigaciones Científicas Licencia Creative Commons 3.0 España (by-nc)
Esta expresión es aplicable a depósitos de arena natural y compacidad relativamente uniforme. La corrección parece provenir de diferencias en la carga última encontrada. Ha sido probada con placas de carga de hasta $1,20 \mathrm{~m}$. Evidentemente, para minimizar los efectos de alteración del Módulo de Balasto debido a las dimensiones del cimiento, interesan placas de carga grandes. Además, si el estrato compresible es de espesor menor que $4 B$ se deben emplear valores de $n$ menores. El autor indica como corrección experimental que, en cimientos rectangulares, es más exacto emplear el lado del cimiento cuadrado de igualárea.

\section{Estimación por ensayo de laboratorio o in situ}

[17] $A C I-336.2 R-88:$ en el apartado 3.3.2 indica que -traducción del autor-:

Para obtener el asiento $\Delta H 21$ seprocederá por integración numérica de la deformación unitaria a lo largo de la profundidad de influencia, calculando posteriormente $\mathrm{K}_{\mathrm{s}}$ como $q / \Delta H$.

Se deben usar varios valores de deformación en la profundidad de influencia, aproximadamente $4 B$, donde $B$ es la mayor dimensión de la base del cimiento.

Los valores de los parámetros elásticos determinados en laboratorio son fuertemente dependientes de las perturbaciones de la muestra y del tipo y calidad de los resultados del análisis triaxil.

A continuación se señalan una serie de correcciones propuestas por distintos autores. Se ha mantenido la diferenciación recogida en las publicaciones entre Módulo Edométrico, $E_{0}$, y el Módulo de Compresibilidad, $E_{s}$. Ambos dependen del valor de tensión que se escoja como valor inicial en el ensayo. Se definen como [31]

$$
E_{m}=\frac{\Delta . \sigma^{\prime}}{\epsilon} \quad E_{0}=\frac{\Delta . \sigma^{\prime}}{-\frac{\Delta V}{V}}
$$

3.a. A partir de Módulo de Compresibilidad del Suelo: Según DE BEER [32]:

1. Cimentación Circular de área $A$ y rigidez suficiente para distribución uniforme de presiones:

$$
K=1,392 \cdot \frac{E_{s}}{\sqrt{A}}
$$

2. Cimentación Circular de área $A$ y rigidez infinitamente grande:

$$
K=1,50 \cdot \frac{E_{s}}{\sqrt{A}}
$$


3. Cimentación Rectangular $a>b$ y rigidez suficiente para distribución uniforme de presiones:

$$
K=1,330 \frac{E_{s}}{\sqrt[3]{a \cdot b^{2}}}
$$

4. Cimentación Rectangular $a>b$ con rigidez propia tan exigua que se produce levantamiento:

$$
K=1,330 \frac{E_{s}}{\sqrt[3]{l^{\prime} \cdot b^{2}}}
$$

si el levantamiento $\alpha . a>\pi$, se denota por $\alpha^{\prime}=\pi / \alpha$, siendo

$$
\alpha=\sqrt[4]{\frac{k_{s} \cdot b}{4 \cdot E \cdot I}}
$$

DIMITROV[32]:

$$
K=\rho \frac{E_{s}}{b\left(1-\mu^{2}\right)},
$$

donde $\rho$ es un Coeficiente de Forma de la Cimentación Rectangular, según la tabla 1.

$\mathrm{Y} \mu$ depende del tipo de terreno de forma tal que:

$0,125 \leq \mu \leq 0,50$ Arenosos y guijarrosos

$0,200 \leq \mu \leq 0,40$ Arcillosos

DIN 4019: [32] $\quad K=\frac{\boldsymbol{E}_{s}}{\boldsymbol{b} \cdot f_{(s, o)}}$,

donde la función $f$ depende de las relaciones $a / b$ y $z / b$, donde $z$ es el espesor de la capa de terreno efectivo, tomándose un valor $z=2 \cdot b$ si el espesor efectivo supera $2 \cdot b$. (tomado de la referencia [32]) (tabla 2).

3.b. A partir de Módulo de Deformación o del Módulo Edométrico Equivalente, $\mathbf{E}_{0}$ del Suelo:

$\operatorname{VOGT}[33]:$

1. Cimentación Circular de área $A$ y rigidez suficiente para distribución uniforme de presiones:

$$
K=1,392 \cdot \frac{E_{0}}{\sqrt{A}}
$$

2. Cimentación Rectangular $a>b$ y rigidez suficiente para distribución uniforme de presiones:

$$
K=1,330 \frac{E_{0}}{\sqrt[3]{a \cdot b^{2}}}
$$

-Nótese la coincidencia con dos de las expresiones propuestas por De Beer-.

\section{KOGLER YSCHEIDING [33]:}

1.Superficie infinita, con capa compresible de espesor $H$ :

(c) Consejo Superior de Investigaciones Científicas

Licencia Creative Commons 3.0 España (by-nc)

$$
K=\frac{E_{0}}{H}
$$

2.Carga en faja de ancho $B$, con capa compresible de espesor $H$ :

$$
K=2 \frac{E_{0}}{B} \frac{1}{\operatorname{Ln}\left(\frac{B+2 H}{B}\right)} \approx \frac{E_{0}}{B}
$$

3.Para superficies circulares o cuadradas, de diámetro o lado $d$ :

$$
K=E_{0} \frac{d+H}{d \cdot H}
$$

relaciones que se han obtenido a partir de un reparto de tensiones por ensanche lineal a $45^{\circ}$.

VESIC (1971) [34]: Para una gran viga sobre profundidad infinita de suelo con módulo de elasticidad o de deformación constantes, el valor de $k$ resulta ser

$$
k_{c}=0,65 \sqrt[12]{\frac{E_{s} \cdot b^{4}}{E_{v} \cdot I_{v}}} \cdot \frac{E_{s}}{1-v^{2}}
$$

$A C I-336.2 R-88$ [17]:

1. Bowles (1982) [24]:

$$
k=\frac{E_{s}}{b\left(1-\mu^{2}\right) I_{w}}
$$

2. Corrección de Comentarios,

$$
k=\frac{E_{s}}{b\left(1-\mu^{2}\right) I_{w} \cdot I_{F}}
$$

donde $I_{w}$ representa el Factor de forma de la base, dependiente de la forma y flexibilidad de la cimentación, e $I_{F}$ denota el factor de empotramiento por la profundidad.

\section{Uso mixto:}

$A C I-336.2 R-88$ [17]:

Úsese uno de los métodos anteriores para estimar el Módulo de Balasto, pero, además, considérese la respuesta del subsuelo a las condiciones de carga dependiente del tiempo.

Propuestas de Métodos Iterativos -recogidos por la norma-:

Úlrich (1988) [35].

Banavalkar y Ulrich (1984) [36].

Focht et al. (1978) [37]

http://informesdelaconstruccion.revistas.csic.es 
TABLA 1

\begin{tabular}{|l|l|l|l|l|l|l|l|l|l|}
\hline a:b & 1,00 & 1,50 & 2,00 & 3,00 & 5,00 & 10 & 20 & 30 & 50 \\
\hline$\rho$ & 1,05 & 0,87 & 0,78 & 0,66 & 0,54 & 0,45 & 0,39 & 0,33 & 0,30 \\
\hline
\end{tabular}

TABLA2

\begin{tabular}{|c|c|c|c|c|c|c|c|}
\hline$z: b \backslash 1: b$ & 1,00 & 1,50 & 2,00 & 3,00 & 5,00 & 10,00 & 20,00 \\
\hline 0,20 & 0,18 & 0,18 & 0,18 & 0,19 & 0,19 & 0,19 & 0,19 \\
\hline 0,40 & 0,29 & 0,31 & 0,32 & 0,33 & 0,33 & 0,34 & 0,34 \\
\hline 0,60 & 0,37 & 0,40 & 0,42 & 0,44 & 0,45 & 0,46 & 0,46 \\
\hline 0,80 & 0,44 & 0,47 & 0,50 & 0,53 & 0,56 & 0,57 & 0,57 \\
\hline 1,00 & 0,49 & 0,53 & 0,57 & 0,61 & 0,64 & 0,67 & 0,67 \\
\hline 1,50 & 0,58 & 0,65 & 0,70 & 0,75 & 0,81 & 0,86 & 0,87 \\
\hline 2,00 & 0,64 & 0,72 & 0,78 & 0,85 & 0,93 & 1,00 & 1,03 \\
\hline 3,00 & 0,70 & 0,82 & 0,89 & 0,99 & 1,09 & 1,20 & 1,25 \\
\hline 5,00 & 0,76 & 0,90 & 1,00 & 1,13 & 1,27 & 1,43 & 1,54 \\
\hline 7,00 & 0,79 & 0,94 & 1,05 & 1,20 & 1,37 & 1,55 & 1,68 \\
\hline 10,00 & 0,81 & 0,97 & 1,09 & 1,26 & 1,45 & 1,68 & 1,85 \\
\hline 20,00 & 0,82 & 0,98 & 1,12 & 1,32 & 1,57 & 1,89 & 2,12 \\
\hline
\end{tabular}

\section{CORRECCIONES AL MÓDULODE BALASTO}

\section{- J. Hann [32], señala}

- Problemas de infravaloración de asientos: calcúlense con los valores límite

- El valor menor de $K$ para el cálculo de presiones sobre el terreno.

- El valor mayor de $K$ para el cálculo de esfuerzos sobre la cimentación.

- Consolidación en los Bordes de una Viga Flotante: implica aumento del Módulo de Balasto.

Experiencias señaladas por Borowika y De Beer demostraron teóricamente que la presión sobre una zapata circular con carga uniformemente repartida (Borowika) $y$ rectangular con carga puntual en el centro ejercen un reparto de presiones que no es en absoluto lineal.

La teoría del semiespacio elástico conduce a la determinación de un coeficiente de balasto variable dentro de la superficie de la cimentación, cualquiera que sea el tipo de ésta.[...] Según Dimitrov [...] cuando la carga está aplicada en un extremo, debe tomarse para $\mathrm{K}$ el doble del valor medio de dicho coeficiente.

Este autor, en la referencia citada, propone unos valores de longitudes elásticas virtuales de las vigas flotantes función de las distintas posiciones de la carga sobre ella. A partir de ellos se han obtenido las correcciones al Módulo de Balasto que considera este autor, en función de la posición relativa de la carga. Estos valores se reflejan en el Gráfico 2

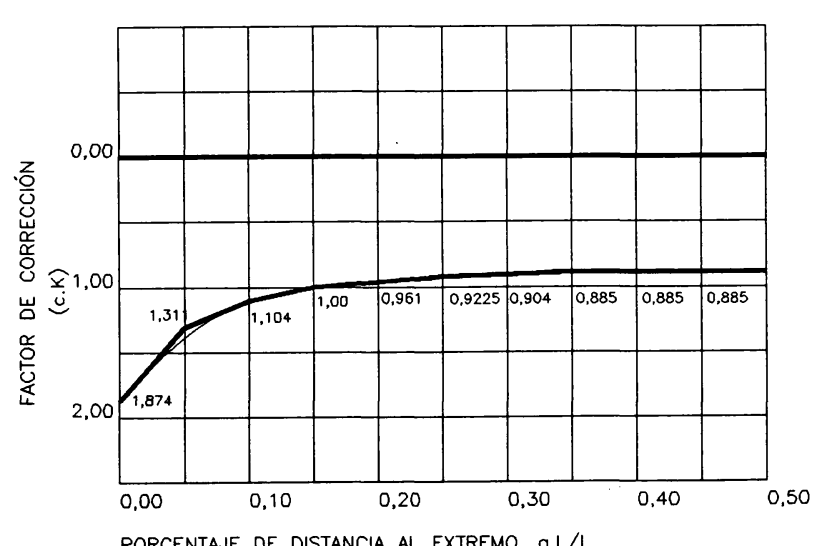

Gráfico 2 
Bowles -[24] [25]- plantea también correcciones por consolidación del terreno. La propia ACI-336 [17], en su apartado 6.9 recomienda: se debe prestar especial atención a los muelles exteriores porque estudios con modelos de gran tamaño han mostrado que se precisa duplicar los muelles finales para conseguir aproximación entre los resultados del cálculo y los experimentales. Esta duplicación produce un mínimo efecto de emparejamiento.

ACI-336.2R-88 [17]-se refiere fundamentalmente al caso de losas de cimentación-:

El uso de un solo Coeficiente de Balasto constante para toda la cimentación puede conducir a errores por infravaloración de los resultados, ya que éste depende de:

- Tamaño del cimiento.

- Área tributaria del nodo.

- Profundidad efectiva.

- Módulo de Balasto elegido.

- Variación del Módulo con la profundidad.

- Características dependendientes del tiempo:

- Asientos por consolidación.

. Asientos por consolidación parcial.

Además la interacción suelo-estructura puede continuar durante largo tiempo hasta que se establezca el equilibrio final entre las cargas sobreimpuestas y las reacciones del terreno soportante.

Factores Detallados (Interacción Suelo-Estructura):

- Tipo del suelo bajo la cimentación.

- Tipo del suelo a grandes profundidades.

- Tamaño de la cimentación.

- Forma de la cimentación.

- Excentricidad de la carga.

- Rigidez de la cimentación.

- Rigidez de la superestructura.

1. Corrección en el Método de Determinación del Módulo:

- Cimientos pequeños $B \leq 1,50 \mathrm{~m}$. Se puede estimar el Módulo de Balasto sobre la base de ensayos de Placa de Carga o sobre la base de las características de los suelos conocidos.

El comportamiento del suelo es generalmente más complicado de lo que se asume en el cálculo de tensiones por medio de la teoría del Módulo de Balasto. Sin embargo, cumpliendo ciertos requisitos y limitaciones, se pueden obtener resultados suficientemente precisos mediante el empleo de estas teorías.

- Cimientos grandes -referido a losas-: estimación por

(c) Consejo Superior de Investigaciones Científicas

Licencia Creative Commons 3.0 España (by-nc) tabulaciones del Módulo de Balasto. La razón estriba en que los efectos de escala resultan demasiado severos. En este sentido, ACI-336 [17], en su apartado 2.2.8, Modulus of Subgrade Reaction, indica que:

Para losas de cimentación, esta propiedad del suelo no puede ser convenientemente estimada con ensayos de campo de placa de carga, porque los efectos de escala son demasiado severos.

Se pueden obtener resultados suficientemente precisos usando la teoría del Módulo de Balasto, pero modificada, para considerar individualmente cargas muertas, cargas vivas, efectos del tamaño, respuestas del terreno asociado...

Se deben considerar zonas de Módulo de Balasto constante de diferente valor para conseguir una estimación más aproximada que la predicha por un único módulo constante. Se puede encontrar una exposición del método en Ball y Notch (1984) y Bowles (1982), así como análisis en Banalvakar y Ulrich (1984) y Focht et al. (1978). [38] [24] [36] [37]

[...] Se debe tener precaución cuando se use el análisis por Elementos Finitos para suelos. Sin unos buenos resultados experimentales, los muelles de terreno derivados de los valores del Módulo de Balasto pueden ser exclusivamente una grosera aproximación a la respuesta actual del suelo. Algunos proyectistas llevan a cabo varios análisis con muelles de terreno, calculados dentro de un rango de Módulos de Balasto para obtener un diseño adecuado.

\section{Correcciones a Adoptar en la Modelización:}

En orden de complejidad decreciente se plantean los siguientes sistemas de modelizaciones:

-Empleo de Coupled Springs

-Sistemas de Emparejamiento Indirecto de Boussinesq, vinculado a estimaciones del asiento por Boussinesq ([17] Sección 6.7).

-Usar en combinación los métodos anterior y siguiente. -Doblar los muelles extremos -Bowles (1974) [23]-Contribución del muelle estimada en proporción lineal a la superficie -práctica común, pero incorrecta-.

Las correcciones a adoptar en la modelización propuestas tratan de conseguir la corrección del comportamiento de muelles aislados que se deforman sin continuidad, estableciendo una deformación continua del modelo de terreno.

\section{FASES DE LA APLICACIÓN DEL MÉTODO[39]}

1. Modelización [de la Estructura,] de la Cimentación y del 
Terreno, de acuerdo con las consideraciones señaladas y las que se realizan en el capítulo específico.

2. Determinación de las Constantes de Muelle que son de aplicación. Implica:

- Cálculo del Módulo de Balasto del Suelo, $k_{s}$ - Cálculo del Módulo de Balasto Corregido del Cimiento, $k_{c}$

- Cálculo del Valor de Muelle teniendo en cuenta las áreas tributarias y las correcciones por consolidación.

\section{Cálculo del Modelo.}

3. Cálculo de las Fuerzas Nodales del Terreno, calculadas como

\section{Fuerza $=$ Muelle de Nodo $\times$ Desplazamiento del Nodo}

Deben calcularse y totalizarse para compararlas con la suma de las fuerzas verticales de la entrada de datos -cargas axiles y peso de la losa- y verificar la validez del cálculo. Debe realizarse como un chequeo de rutina.

4. Presiones sobre el Terreno en los Nodos, calculadas como:

$$
q_{i}=k_{s} x \text { Desplazamiento }
$$

Evidentemente, los valores así calculados deben ser aceptables para el terreno.

De todas formas, como la rigidez axil de la barra ficticia que emula el terreno se expresa como

$$
\frac{E \cdot A}{l}=K \cdot b \cdot x
$$

donde $x$ es el paso de la discretización. Evidentemente, según lo expuesto anteriormente, siendo $x$ constante, resulta que la capacidad de carga del cimiento es muy poco dependiente de su ancho $b$. Se puede corregir este efecto definiendo un ancho equivalente, $b_{\text {eq }}$ que sería el ancho de un cimiento cuadrado de igual área, de forma similar al coeficiente corrector que propone Calavera -[21] pág. 342 y 343- para el caso de arcillas. La importancia del problema se plantea en la capacidad portante de la zapata de medianería, ya que de considerarla funcionando principalmente en un sentido $u$ otro -esto es, planteando qué lado se designa por $a$ y cuál por $b$-, habría una notoria diferencia de la capacidad de carga del cimiento. La consideración anteriomente señalada permite obtener un valor medio para el caso.

Una justificación de esta corrección se puede plantear desde el principio físico del empuje de cuerpos sumergidos. Este método es conocido, como es bien sabido, como de las vigas flotantes, donde se expresa que se considera el cimiento como flotando sobre un líquido. Es sabido que en el caso de los líquidos, el empuje que recibe el cuerpo sumergido es proporcional al peso del volumen de líquido desplazado. En este sentido se justifica la introducción del concepto de Area Equivalente.

\section{CONCLUSIONES}

El avance en los recursos informáticos ha puesto al alcance del técnico el tratamiento conjunto de suelo-cimientoestructura para casos cotidianos, introduciendo un mayor nivel de precisión. El mayor grado de precisión obtenido se refleja principalmente en una serie de aspectos como son

-Leyes de esfuerzos más ajustadas a la realidad.

-Dimensionado de los elementos de cimentación teniendo en cuenta su deformabilidad y una respuesta del terreno más ajustada.

-Estimación afinada de las presiones realmente transmitidas al terreno.

- Posibilidades de estudio de asientos.

Empero, el primer condicionante que surge es conseguir una modelización del suelo adecuada. En numerosos casos, este problema se ha resuelto recurriendo al método del módulo de balasto, bien conocido y único del que se posee contraste práctico.

Desgraciadamente, las referencias que los manuales de los programas informáticos ofrecen sobre este aspecto son muy escasas, siendo sin embargo un problema trascendente para la consecución de un modelo afinado. Las referencias que se incluyen en el artículo permiten decidir sobre la validez del modelo para cada caso particular, así como también la elección de valores adecuados para el módulo, de acuerdo con el tipo de terreno y de cimientos -así como de sus dimensiones- que se encuentren involucrados en el problema.

Por último, se recogen una serie de refinamientos que diversos autores han ido proponiendo para la mejora del modelo y que pueden ser fácilmente implementados en sistemas informáticos, obteniendo unos grados de precisión de nivel superior.

\section{BIBLIOGRAFÍA}

[1] Véase, por ejemplo, CALAVERA, J., Cálculo de Estructuras de Cimentación, Capítulo 3. INTEMAC, $\operatorname{Madrid}\left(2^{\mathrm{a}} \mathrm{Ed}\right.$.)

[2] Véase, por ejemplo, CALAVERA, J., Cálculo de Flechas en Estructuras de Hormigón Armado, Capítulo 1. INTEMAC, Madrid, 1992. 
[3] TERZAGHI, KARL Y PECK, RALPH B., Soil Mechanics in Engineering Practice. Whiley, New York, 1967. Versión Española Mecánica de Suelos en la Ingeniería Práctica. Editorial El Ateneo S.A. $1^{\text {a }}$ Ed: $1955.2^{\mathrm{a}}$ Ed.: 1973. págs. 279 y ss. / 484 y ss.

[4] JIMÉNEZ SALAS, Geotecniay Cimientos. Editorial Rueda. Madrid, $1980\left(2^{\mathrm{a}} \mathrm{Ed}\right.$. $)$

[5] JIMÉNEZ SALAS, Geotecnia y Cimientos, Tomo III, Capítulo 1. Editorial Rueda. Madrid, 1980 (2 Ed.)

[6] TERZAGHI, KARL, Evaluation of Coefficients of Subgrade Reaction. Geotecnique, 1955, Vol. 5, n1 4, pp. 297-326.

[7] Dr. CHRISTIAN, JOHN T., Soil-Foundation-Structure Interaction. Proceedings, Short Course-Seminar on Analysis and Design of Buildings Foundations. Lehigh University. Envo Press, Lehigh Valley, 1976, pp. 149-179.

[8] Whitman, R.; Casagrande, D.; Karstrud, Kjell y Simon, R., Performance of Foundation for Altair Radar. ASCE, journal of Soil Mechanics and Foundation Division, Vol.97, enero, 1971, pp 1-18.

[9] De SIMONE, S.V. Y GOULD, J.P., Performance of two mat foundations on Boston blue clay. Presentada a ASCE Annual and National Environmental Engineering Meeting. St. Louis, octubre 1971.

[10] WINKLER, E., Die Lehre von Elastizität und Festigkeit (Sobre Elasticidad y Resistencia). Praga, 1867, pp. 182.

[11] ZIMMERMANN, H., Die Berechnung des Eisenbahn Oberbaues. (Cálculo de superestructuras de vías de ferrocarril)Berlín, 88

[12] D. GRAUX, Fundamentos de Mecánica del Suelo Proyecto de Muros y Cimentaciones.

Editores Técnicos Asociados, Barcelona, 1975 (2ªd.)

[13] GIBSON, R. E., Some Results Concerning Displacements in a Non-Homogenous Elastic Half-Space. Geotechnique, Vol. 17, pp., 58-67, 1967 y The analytical method in soil mechanics. Geotechnique, Vol. 21, pp., 115-140, 1974 (Referencias tomadas de Jiménez Salas, Geotecnia y Cimientos, Tomo III, Cap. 1. pág. 4 -Editorial Rueda, Madrid, 1980 ( $2^{\mathrm{a}}$ Ed.)- que se refiere a Vol.2, Pág. 265 y ss.)

[14] JIMÉNEZ SALAS., Geotecnia y Cimientos, Vol. II. Cap., pág. 265. Editorial Rueda. Madrid, $1980\left(2^{\mathrm{a}} \mathrm{Ed}\right.$.)

[15] MASAAL'SKII, E.K., Experimental Study of a Flexible Beam on a Sand Foundation. Soil Mechanics and Foundation Engineering, Santiago de Chile, 1964, Vol II, pp 71-86.

[16 WARD, W.H.; BURLAND, J.B. Y GALLOIS, R.W., Geotechnical Assessment of a Site at Mundford, Norfolk for a Large Proton Accelerator. Geotechnique, 1968, Vol 18 pp. 399-431.

[17] ACI 336 2R-88. Suggested Analysis Design Procedures for Combined Footings and Mats.
[18] HORVATH, J. S., A Study of analytical methods for determining the response of mat foundations to static loads. Tesis Doctoral del Instituto Politécnico de Nueva York, 1979.

[19] Referencia tomada de [12], pág. 309. Si bien no aparece referida en ésta, parece corresponder a Caquot y Kerisel, Traité de mécanique des sols. París, 1956.

[20] Sopeña, L., Jornadas sobre Problemática Geotécnica en la Edificación. Cimentaciones, pag. 64 y ss. Lugo, junio 1994.

[21] CALAVERA, J., Estructuras de Cimentación. INTEMAC, Madrid (20 Ed.).

[22] L'HERMINIER, Étude des fondations de surface. Annales del I.T.B.T.P. Enero, 1957. Sols et Fondations n1 24

[23] BOWLES, J.E., Analytical and Computer Methods in Foundation Engineering. McGraw-Hill BookCo., New York, 1974,pp.154-170.

[24] BOWLES, J.E., Foundation Analysis and Design. McGraw-Hill Book Co., New York, 1982, 800 pp. (3 ${ }^{\text {a }}$ Ed.)

[25] BOWLES, J.E., Physical and Geotechnical Properties of Soil. McGraw-Hill Book Co., New York, $1984\left(2^{\mathrm{a}}\right.$ Ed.)

[26] Dept. of Navy. Soil Mechanics. Design Manual NAVFAC DM-7.1. Naval Facilities Engineering Command, Alexandria pp. 7.2-129 a 7.2-175.

[27] KRAMRISH, F., Footings. Handbook of Concrete Engineering.Mark Fintel,Editor, Van Nostrand Reinhold Co. New York, 1984. pp. 139-168 (2 Ed.)

[28] CALAVERA, J., Estructuras de Cimentación, Tabla GT-9. INTEMAC, Madrid ( $2^{\mathrm{a}}$ Ed.)

[29] JIMÉNEZ SALAS, Geotecniay Cimientos III.Capítulo 1. Tabla 1.1. Editorial Rueda. Madrid, 1980 (2ª Ed.)

[30] SOWERS, G.F., Foundation Modulus for a Mat on Sand. Preprint $\mathrm{n} 1$ 2937. American Society of Civil Engineers, oct 17-21. New York, 1977.

[31] JIMÉNEZ SALAS. Geotecnia y Cimientos, Vol. I, págs. 181 y 320 . Editorial Rueda. Madrid, $1980\left(2^{\mathrm{a}} \mathrm{Ed}\right.$.)

[32] Tomado DE J. HANN, Vigas Continuas, Pórticos, Placas y Vigas Flotantes sobre Terreno Elástico. Editorial Gustavo Gili, $3^{\text {a }}$ Ed. págs. 334 y ss.

[33] Tomado de JIMÉNEZ SALAS,Geotecnicay Cimientos III. Capítulo 1. Editorial Rueda. Madrid, $1980\left(2^{2} \mathrm{Ed}\right.$.)

[34] VESIC, ALEKSANDER, Beams on Elastic Subgrade and the Winkler's Hypothesis. $5^{\text {th }}$ International Conference ofSoilMechanicsandFoundationEngineering. París, 1971, Vol. I. pp. 845-850.

http://informesdelaconstruccion.revistas.csic.es 
[35] ULRICH, E.J., Geotechnical Considerations in Mat Foundations Design. Structural and Geotechnical Lecture Series. ASCE, Sección Illinois. Chicago, 1988

[36] BANAVAKAR P.V. Y ULRICH, E.J., Republic Bank Center: Structural and Geotechnical Features. International Conference on Tall Buildings. Singapur, Octubre, 1984.

[37] FOCHT J.A.; KHAN, F.R. Y GEMEINHARDT, J.P., Performance of One Shell Plaza Deep Mat Foundation. Proceedings ASCE, V. 104, Mayo, 1978. pp. 593-608.
[38] BALL, S.C. Y NOTCH, J.S., Computer Analysis/Design of Large Mat Foundations. Journal of Structural Engineering ASCE, Vol.1 10n1 5. mayo, 1984.pp. 11801196.

[39] Se puede consultar la exposición realizada en PÉREZ VALCÁRCEL et alli Estructuras de Hormigón Armado. Tórculo Artes Gráficas, Santiago de Compostela, 1994.

\section{Publicaciones del Instituto Eduardo Torroja-CSIC}

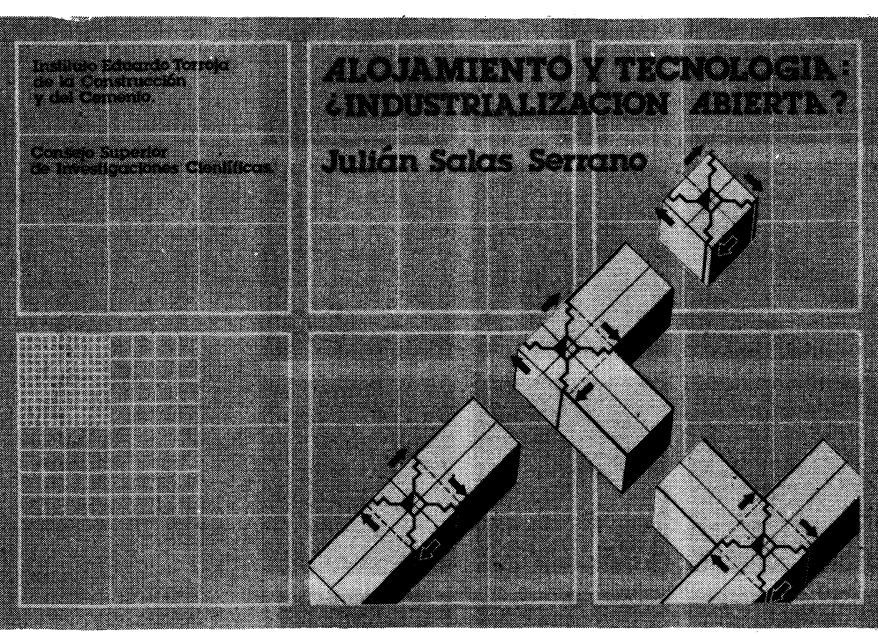

ALOJAMIENTO Y TECNOLOGIA: ¿INDUSTRIALIZACION ABIERTA?

JULIAN SALAS, ING. IND. (I.E.T.c.c.)

Un volumen de 160 páginas, 109 figuras y 16 tablas. Tamaño $240 \times 168 \mathrm{~mm}$. Encuadernado en rústica.

\section{SUMARIO:}

Prólogo Prof. G. Ciribini.

\section{Introducción}

Capítulo 1.- La industrialización en las proclamas y manifiestos de arquitectura.

Capítulo 2.- ¿Réquiem por la construcción industrializada?

Capítulo 3.-Algunos conceptos básicos.

Capitulo 4.-¿Proyecto tradicional, construcción industrializada?

Capitulo 5. - Componentes.

Capítulo 6. - La coordinación dimensional hoy.

Capítulo 7.-Flexibilidad, intercambiabilidad y catálogos.

Capítulo 8. - Industrialización, normativa y calidad.

Capitulo 9.-Reflexiones finales.

publicación del

INSTITUTO EDUARDO TORROJA 